\title{
An Inverted Pendulum Model Describing the Lateral Pedestrian-Footbridge Interaction
}

\author{
Bin Zhen $\left(\mathbb{D},{ }^{1}\right.$ Liang Chang $\mathbb{D}^{2}{ }^{2}$ and Zigen Song ${ }^{3}{ }^{3}$ \\ ${ }^{1}$ School of Environment and Architecture, University of Shanghai for Science and Technology, Shanghai 200093, China \\ ${ }^{2}$ School of Civil Engineering and Architecture, Nanchang Hangkong University, Nanchang, Jiangxi 330063, China \\ ${ }^{3}$ College of Information Technology, Shanghai Ocean University, Shanghai 201306, China
}

Correspondence should be addressed to Zigen Song; zigensong@163.com

Received 3 June 2018; Revised 19 September 2018; Accepted 27 September 2018; Published 1 November 2018

Academic Editor: Angelo Di Egidio

Copyright () 2018 Bin Zhen et al. This is an open access article distributed under the Creative Commons Attribution License, which permits unrestricted use, distribution, and reproduction in any medium, provided the original work is properly cited.

In this paper, the lateral pedestrian-footbridge interaction is investigated by using the model of an inverted pendulum on a cart. The inverted pendulum and the cart separately represent the synchronous pedestrians and the footbridge. The pivot point of the inverted pendulum is considered to vibrate harmonically to model the walking motion of the pedestrians. The proposed inverted pendulum model avoids the difficulty of the determination of the lateral force induced by the pedestrians applying to the footbridge, which was usually treated based on a semiempirical approach in previous works. Moreover, the model can describe the whole process: how the lateral amplitude of the bridge increases from small to large. Measurement data showed that a normal pedestrian always keeps the ratio of $1 / 2$ between the lateral and vertical step frequencies. The theoretical analysis for the inverted pendulum model indicates that such walking habit of pedestrians is the root of the frequency-locking phenomenon, which eventually results in excessive lateral vibrations of the bridge. Furthermore, such walking habit also is a key factor in the occurrence of the "jump phenomenon" in the London Millennium Bridge.

\section{Introduction}

The excessive lateral vibrations of footbridges induced by pedestrians have received much concern in the past decade. The lateral vibrations of a footbridge occur due to the zigzag movement of the pedestrians. Measurements showed that a pedestrian will induce vertical and horizontal dynamic time-varying forces at the same time on a rigid surface [1]. The lateral force is about $4 \%$ of the pedestrian's weight, and the frequency is between 0.75 $1.25 \mathrm{~Hz}$ [2]. Observations showed that many footbridges with excessive lateral vibrations have a lateral natural frequency near $1 \mathrm{~Hz}$, such as the London Millennium Bridge, the T-bridge, and M-bridge in Japan [3]. If a footbridge has a lateral mode of vibration with a natural frequency within the range of the lateral walking frequency, the direct resonance will be achieved to result in excessive lateral vibrations.

More complex cause, the internal resonance mechanism [4], has been proposed to explain the excessive lateral vibrations of footbridges. If the bridge is characterized by a 2:1 ratio between vertical-mode and lateral-mode frequencies and its vertical mode satisfies the direct resonance conditions, energy can flow between the vertical and lateral modes because of the structural nonlinearities [5]. However, both the direct resonance and the internal resonance theories have difficulty in explaining the frequency-locking phenomenon between pedestrians and a footbridge in the lateral direction.

Dynamic interaction mechanisms have got special concern in the past few years. The interactive force between pedestrians and a footbridge is the key factor to precisely describe the dynamics of the bridge. Dallard et al. [6] assumed that the external lateral force induced by pedestrians is proportional to the lateral velocity of the bridge to explain the excessive lateral vibration on the London Millennium Bridge. Dallard's model provides an instability threshold, which has been adopted in the engineering practice. Nevertheless, this model indicates that the bridge response will increase infinitely when the lateral force is large enough. 
To avoid the irrationality, Nakamura and Kawasaki [2, 3] modified Dallard's model by assuming that the lateral force induced by pedestrians is proportional with the bridge velocity only at low velocities. Nakamura's model allows schematization of self-limiting nature when the bridge velocity becomes large. Roberts [7] considered that synchronization between pedestrians and a footbridge occurs when the displacement of the footbridge is greater than that of the pedestrians, and the interactive force harmonically varies with frequency of about $1 \mathrm{~Hz}$. Macdonald [8] used an inverted pendulum model from the biomechanics field to estimate the lateral force induced by a walking pedestrian. However, the bridge motion is absent in the model; that is, dynamical interaction between pedestrians and the bridge has not been fully considered in this estimation. Up to now [9], the lateral force exerted by pedestrians was generally tackled based on an empirical approach, especially based on the measurement data. Experimental measurements [10] showed that the lateral force induced by a pedestrian walking on stationary platforms is approximately harmonic. But, such approximation may not be appropriate if the pedestrian walks on a footbridge with a laterally large amplitude. Experimental measurements involving pedestrians walking on a moving platform [6] showed that the lateral force induced by pedestrians is a function of the deck motion amplitude. Piccardo and Tubino [5] considered that the dynamic loading factor can be approximately expressed as a linear function of the deck motion amplitude. Based on the assumption, Piccardo et al. explained why the London Millennium Bridge has a lateral vibration with a frequency of $0.48 \mathrm{~Hz}$. Ingólfsson et al. [11] found that the lateral force induced by a pedestrian is a summation of the equivalent static load that represents the lateral force exerted by a pedestrian on a rigid surface and the motion-induced force that is attributed to the movement of the structure. In their model, it is a frequency- and amplitude-dependent lateral force.

From the literature above, it is worth looking at two aspects of the study of the dynamic interaction between pedestrians and a footbridge. One is that almost all the lateral force models are established according to measurement data and plausible rational assumptions; Strict and exact mathematical models are not available yet. The other is that the existing lateral force models lack the description of the changing process when the lateral amplitude of the bridge increases from small to large. For small lateral vibrations of the bridge, the lateral force is considered to be harmonic; with the increase of the lateral amplitude, the lateral force is a function of the lateral amplitude or velocity of the bridge, or has a more complex relation with some characteristic quantities of the bridge. When pedestrians begin to walk on a stationary bridge, no one knows the full dynamic interaction process: how the bridge increases its lateral amplitude under pedestrians excitation. This might be a key point why some observations on footbridges cannot be rationally explained. For example, the "jump phenomenon" in the London Millennium Bridge is curious anomaly [12]. It is still confused to understand why the bridge amplitude decreased suddenly after a sudden increase while the pedestrians did not significantly reduce their walking speed. To attempt to solve the questions mentioned above, we used an inverted pendulum on a cart to model the lateral interaction between pedestrians and a footbridge. The inverted pendulum represents the synchronization pedestrians, while the cart, the bridge. To model the pedestrians' walking motion on the bridge, we consider the inverted pendulum has harmonica vibrations at its pivot point. The proposed model in this paper avoids the difficulty of the determination of lateral force induced by pedestrians. Furthermore, the model provides a possible way to understand the changing process: how the lateral amplitude of the bridge increases from small to large. Our theoretical analysis for the model shows that some walking habits of the pedestrians are the key factor to result in the frequency-locking phenomenon. The habit also is the reason why the lateral amplitude of the London Millennium Bridge suddenly decreased while the pedestrians did not reduce their walking speed.

The rest of the paper is organized as follows: the governing equation of the inverted pendulum model is given in Section 2; asymptotic solutions of the model are discussed in Section 3, and the resonance conditions for the inverted pendulum system are derived. In Section 4 , the stability of the inverted pendulum system is investigated to illustrate the inevitability of the resonance for some footbridges. In Section 5, numerical simulations are carried out to demonstrate that the proposed inverted pendulum model is able to give quantitative results. Conclusions are drawn in Section 6.

\section{The Model Equation}

Consider the inverted pendulum of length $L$ and lump mass $m$ on a cart of mass $M$, shown as in Figure 1. The inverted pendulum represents the synchronization pedestrians, and the cart represents a footbridge with a stiffness $k$. Here, we omit the damping. To analyze the lateral pedestrianfootbridge interaction, we assume that the pivot point of the inverted pendulum vibrates harmonically along the $y$-axis to model the synchronization pedestrians' walking motion. Under such excitation, the cart sways along the $x$-axis, which simulates the lateral vibrations of the footbridge. The dynamics of the lateral pedestrian-footbridge interaction can be discussed as long as the inverted pendulum is stable.

Denote the angle of the pendulum with respect to the vertical direction by $\theta$ and the gravitational acceleration by g. The inclination angle $\theta$ is defined such that it is negative in the position shown in Figure 1. The governing equation of the inverted pendulum model can be derived by using Lagrange's equations. The positions of the lump mass $m$ and the cart are written as

$$
\begin{aligned}
& m:(x+L \sin (\theta), y+L \cos (\theta)), \\
& M:(x, 0),
\end{aligned}
$$

where $x$ and $y$ represent the horizontal and vertical displacements of the pivot point of the inverted pendulum, 


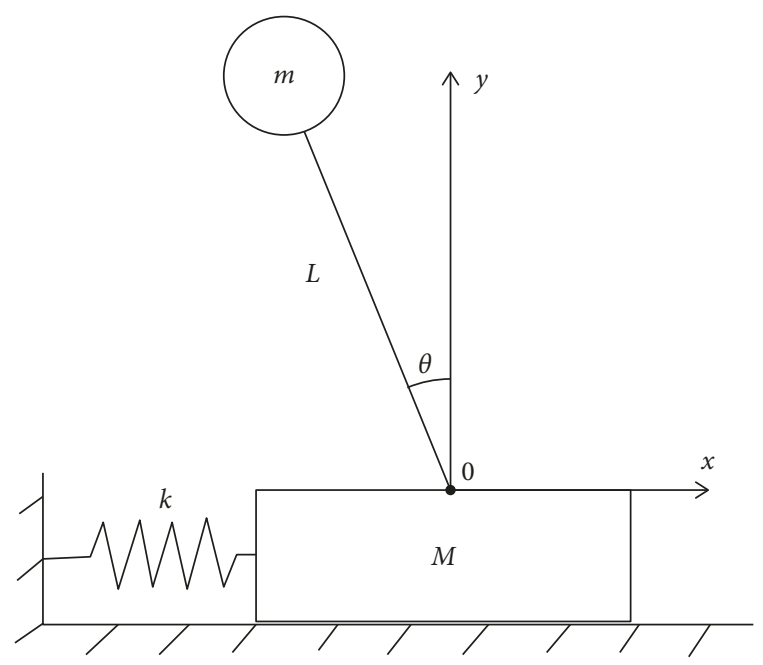

FIGURE 1: Sketch of the inverted pendulum on a cart.

respectively. Then, the kinetic and potential energy are separately expressed by

$$
\begin{aligned}
& T=\frac{1}{2} m\left[(\dot{x}+L \dot{\theta} \cos (\theta))^{2}+(\dot{y}-L \dot{\theta} \sin (\theta))^{2}\right]+\frac{1}{2} M \dot{x}^{2}, \\
& V=m g(y+L \cos (\theta))+\frac{1}{2} k x^{2},
\end{aligned}
$$

where $\cdot$ denotes the derivative versus time.

The Lagrangian of the inverted pendulum system is given by

$$
H=T-V,
$$

which should satisfy the following Lagrange equations:

$$
\begin{aligned}
& \frac{d}{d t} \frac{\partial H}{\partial \dot{\theta}}-\frac{\partial H}{\partial \theta}=0 \\
& \frac{d}{d t} \frac{\partial H}{\partial \dot{x}}-\frac{\partial H}{\partial x}=0
\end{aligned}
$$

Substituting Equations (2) and (3) into Equation (4) and neglecting the nonlinear terms of the position and velocity of the inverted pendulum and cart, the model equation can be written as

$$
\begin{aligned}
\ddot{\theta}+\frac{1}{L} \ddot{x}-\frac{1}{L}(\ddot{y}+g) \theta & =0, \\
\ddot{x}+\frac{m L}{M+m} \ddot{\theta}+\frac{k x}{M+m} & =0 .
\end{aligned}
$$

Assume that the pivot point has harmonic vibrations with the form of $y=A \cos (\omega t)$, where $A$ and $\omega$ represent the amplitude and frequency of vibrations. In addition, let time be normalized according to the transformation $\omega t \longrightarrow t$. Then, Equation (5) can be rewritten as

$$
\begin{aligned}
\ddot{\theta}+\beta_{1} \ddot{x}+(\epsilon \cos (t)+\delta) \theta & =0, \\
\ddot{x}+\beta_{2} \ddot{\theta}+\beta_{3} x & =0,
\end{aligned}
$$

where

$$
\begin{aligned}
& \beta_{1}=\frac{1}{L}, \\
& \beta_{2}=\frac{m L}{M+m}, \\
& \beta_{3}=\frac{k}{(M+m) \omega^{2}}, \\
& \epsilon=\frac{A}{L}, \\
& \delta=-\frac{g}{L \omega^{2}} .
\end{aligned}
$$

According to physical meanings of parameters, $0<\epsilon<1$ and $-1 \ll \delta<0$. In this paper, we will analyze the stability conditions of system (6) to understand the dynamics of the pedestrian-footbridge interaction. We first find the resonance conditions of Equation (6) in the next section.

\section{Resonance Conditions for System (6)}

We first find the solution of Equation (6) with $0<\delta<1$, which has the following form:

$$
\begin{aligned}
& \theta=\theta_{0}+\epsilon \theta_{1}+\epsilon^{2} \theta_{2}, \cdots, \\
& x=x_{0}+\epsilon x_{1}+\epsilon^{2} x_{2}, \cdots .
\end{aligned}
$$

Substituting Equation (8) into Equation (6) and equating the coefficients of each power of $\epsilon$ to be zero, one has

$$
\begin{aligned}
\ddot{\theta}_{0}+\delta \theta_{0}+\beta_{1} \ddot{x}_{0} & =0, \\
\ddot{x}_{0}+\beta_{2} \ddot{\theta}_{0}+\beta_{3} x_{0} & =0, \\
\ddot{\theta}_{1}+\delta \theta_{1}+\beta_{1} \ddot{x}_{1} & =-\cos (t) \theta_{0}, \\
\ddot{x}_{1}+\beta_{2} \ddot{\theta}_{1}+\beta_{3} x_{1} & =0 .
\end{aligned}
$$

Equations (9) and (10) can be separately rewritten as

$$
\begin{aligned}
\frac{d^{4} \theta_{0}}{d t^{4}}+\frac{\delta+\beta_{3}}{1-\beta_{1} \beta_{2}} \ddot{\theta}_{0}+\frac{\delta \beta_{3}}{1-\beta_{1} \beta_{2}} \theta_{0} & =0, \\
x_{0} & =\frac{1-\beta_{1} \beta_{2}}{\beta_{1} \beta_{3}} \ddot{\theta}_{0}+\frac{\delta}{\beta_{1} \beta_{3}} \theta_{0},
\end{aligned}
$$

$$
\begin{aligned}
\frac{d^{4} \theta_{1}}{d t^{4}}+\frac{\delta+\beta_{3}}{1-\beta_{1} \beta_{2}} \ddot{\theta}_{1}+\frac{\delta \beta_{3}}{1-\beta_{1} \beta_{2}} \theta_{1}= & \frac{\left(1-\beta_{3}\right) \theta_{0}-\ddot{\theta}_{0}}{1-\beta_{1} \beta_{2}} \cos (t) \\
& +\frac{2 \dot{\theta}_{0}}{1-\beta_{1} \beta_{2}} \sin (t), \\
x_{1}= & \frac{1-\beta_{1} \beta_{2} \ddot{\theta}_{1}}{\beta_{1} \beta_{3}} \\
& +\frac{\delta \theta_{1}+\cos (t) \theta_{0}}{\beta_{1} \beta_{3}} .
\end{aligned}
$$

The general solution of Equation (11) is given by 


$$
\begin{aligned}
\theta_{0}= & a_{1} \cos \left(\gamma_{1} t\right)+a_{2} \sin \left(\gamma_{1} t\right)+a_{3} \cos \left(\gamma_{2} t\right)+a_{4} \sin \left(\gamma_{2} t\right), \\
x_{0}= & \mu_{1}\left[a_{1} \cos \left(\gamma_{1} t\right)+a_{2} \sin \left(\gamma_{1} t\right)\right] \\
& +\mu_{2}\left[a_{3} \cos \left(\gamma_{2} t\right)+a_{4} \sin \left(\gamma_{2} t\right)\right],
\end{aligned}
$$

where $a_{i}$ are constants dependent on the initial conditions, and

$$
\begin{aligned}
& \mu_{1}=\frac{\delta-\left(1-\beta_{1} \beta_{2}\right) \gamma_{1}^{2}}{\beta_{1} \beta_{3}}, \\
& \mu_{2}=\frac{\delta-\left(1-\beta_{1} \beta_{2}\right) \gamma_{2}^{2}}{\beta_{1} \beta_{3}}, \\
& \gamma_{1}=\frac{\sqrt{2}}{2} \sqrt{\frac{\delta+\beta_{3}}{1-\beta_{1} \beta_{2}}+p}, \\
& \gamma_{2}=\frac{\sqrt{2}}{2} \sqrt{\frac{\delta+\beta_{3}}{1-\beta_{1} \beta_{2}}-p}, \\
& \gamma_{2}=\frac{\sqrt{\delta^{2}+2 \delta \beta_{3}\left(2 \beta_{1} \beta_{2}-1\right)+\beta_{3}^{2}}}{1-\beta_{1} \beta_{2}} .
\end{aligned}
$$

Substituting Equation (13) into Equation (12), the particular solution of Equation (12) can be expressed by

$$
\begin{aligned}
\theta_{1}= & a_{1}\left[\alpha_{1} \cos \left(\left(\gamma_{1}-1\right) t\right)+\alpha_{2} \cos \left(\left(\gamma_{1}+1\right) t\right)\right] \\
& +a_{2}\left[\alpha_{1} \sin \left(\left(\gamma_{1}-1\right) t\right)+\alpha_{2} \sin \left(\left(\gamma_{1}+1\right) t\right)\right] \\
& +a_{3}\left[\alpha_{3} \cos \left(\left(\gamma_{2}-1\right) t\right)+\alpha_{4} \cos \left(\left(\gamma_{2}+1\right) t\right)\right] \\
& +a_{4}\left[\alpha_{3} \sin \left(\left(\gamma_{2}-1\right) t\right)+\alpha_{4} \sin \left(\left(\gamma_{2}+1\right) t\right)\right], \\
x_{1}= & v_{1}\left[a_{1} \cos \left(\left(\gamma_{1}-1\right) t\right)+a_{2} \sin \left(\left(\gamma_{1}-1\right) t\right)\right] \\
& +v_{2}\left[a_{1} \cos \left(\left(\gamma_{1}+1\right) t\right)+a_{2} \sin \left(\left(\gamma_{1}+1\right) t\right)\right] \\
& +v_{3}\left[a_{3} \cos \left(\left(\gamma_{2}-1\right) t\right)+a_{4} \sin \left(\left(\gamma_{2}-1\right) t\right)\right] \\
& +v_{4}\left[a_{3} \cos \left(\left(\gamma_{2}+1\right) t\right)+a_{4} \sin \left(\left(\gamma_{2}+1\right) t\right)\right],
\end{aligned}
$$

where

$$
\begin{aligned}
\alpha_{1} & =f_{1}\left(\gamma_{1}-1\right), \\
\alpha_{2} & =f_{1}\left(\gamma_{1}+1\right), \\
\alpha_{3} & =f_{1}\left(\gamma_{2}-1\right), \\
\alpha_{4} & =f_{1}\left(\gamma_{2}+1\right), \\
\nu_{1} & =f_{2}\left(\gamma_{1}-1\right), \\
\nu_{2} & =f_{2}\left(\gamma_{1}+1\right), \\
\nu_{3} & =f_{2}\left(\gamma_{2}-1\right), \\
\nu_{4} & =f_{2}\left(\gamma_{2}+1\right), \\
f_{1}(y) & =\frac{\beta_{3}-y^{2}}{2\left[\left(\beta_{1} \beta_{2}-1\right) y^{4}+\left(\delta+\beta_{3}\right) y^{2}-\delta \beta_{3}\right]}, \\
f_{2}(y) & =\frac{1}{\beta_{1} \beta_{3}}\left[\frac{1}{2} \beta_{1} \beta_{3}+f_{1}(y)\left(\delta-y^{2}\left(1-\beta_{1} \beta_{2}\right)\right)\right] .
\end{aligned}
$$

To demonstrate the validity of the first-order perturbation solution, comparison between expressions (13) and (15) and numerical results of Equation (6) is carried out for two different cases, which is presented in Figure 2. Clearly, it has a good agreement between the two approaches, which indicates that the perturbation method is valid to derive the asymptotic solutions of Equation (6). We also show in Figure 3 that the analytical solution will have less degree of coincidence with the numerical simulation results if the value of $\epsilon$ increases. The higher order terms of $\epsilon$ have to be calculated to improve the coincidence between the two approaches.

According to Equation (15), there cannot be any bounded solutions of Equation (6) if the value of the denominator of $f_{1}\left(\gamma_{i} \pm 1\right)$ is equal to zero, that is,

$$
\left(\beta_{1} \beta_{2}-1\right) y^{4}+\left(\delta+\beta_{3}\right) y^{2}-\delta \beta_{3}=0,
$$

where $y=\gamma_{i} \pm 1, i=1,2$. By letting $n=m / M$ in Equation (17), it is easy to check that the critical values of $\delta$ and $\beta_{3}$ are only related to $n$. According to physical meanings of parameters, the mass of the pedestrians is much less than that of the footbridge, that is, $n \ll 1$. The curves defined by Equation (17) with $y=\gamma_{1}-1$ are presented in Figure 4, where $n$ is taken as $0,0.01,0.05$, and 0.1 , respectively.

Clearly, from Figure 4 for a small $\delta>0$, the resonance of system (6) occurs near $\beta_{3}=1 / 4$ and $\beta_{3}=1$. The influence of $n$ on the resonance conditions is small if $n$ varies near zero.

In fact, if $n \longrightarrow 0, \beta_{2} \longrightarrow 0$. The second equation of Equation (6) can be approximately written as

$$
\ddot{x}+\beta_{3} x=0 .
$$

The solution of Equation (18) is given by $x=$ $a_{0} \cos \left(\sqrt{\beta_{3} t}\right)$, where $a_{0}$ is a constant determined by the initial conditions. Substituting this solution into the first equation in Equation (6) yields

$$
\ddot{\theta}+(\epsilon \cos (t)+\delta) \theta=a_{0} \beta_{1} \beta_{3} \cos \left(\sqrt{\beta_{3} t}\right) .
$$

If the right side of Equation (19) is equal to zero, it becomes the classical Mathieu equation. The stability chart of the Mathieu equation is well known [13-15], and the region near $\delta=0$ in the $\epsilon-\delta$ parametric plane is shown in Figure 5.

For parameters on the line $\mathrm{AB}$ in Figure 5, Equation (6) has periodic solutions with period of $4 \pi$ regardless of whether $\delta<0$. Under such case, if $\sqrt{\beta_{3}}=1 / 2,1,3 / 2, \cdots$, no bounded solution can be found in Equation (6). From Equations (18) and (19), it is clear that the resonance in Equation (6) occurs because of periodic solutions of the inverted pendulum with period of near $4 \pi$. From the stability chart in Figure 5, if $\delta<0$, there still exists periodic solutions with period of $4 \pi$ in Equation (6). According to Figure 4, the linear system (6) is affected slightly by $n$ when $n$ is small. Therefore, if $\delta<0$ and $n$ varies near 0 , the unbounded solutions still occur near $\sqrt{\beta_{3}}=1 / 2,1, \cdots$. In practice engineering, we usually only focus on the first resonance condition, and then we emphasize on the condition $\sqrt{\beta_{3}}=1 / 2$.

Recall

$$
\beta_{3}=\frac{k}{(M+m) \omega^{2}}=\left(\frac{\sqrt{k / M+m}}{\omega}\right)^{2}=\left(\frac{1}{2}\right)^{2} .
$$



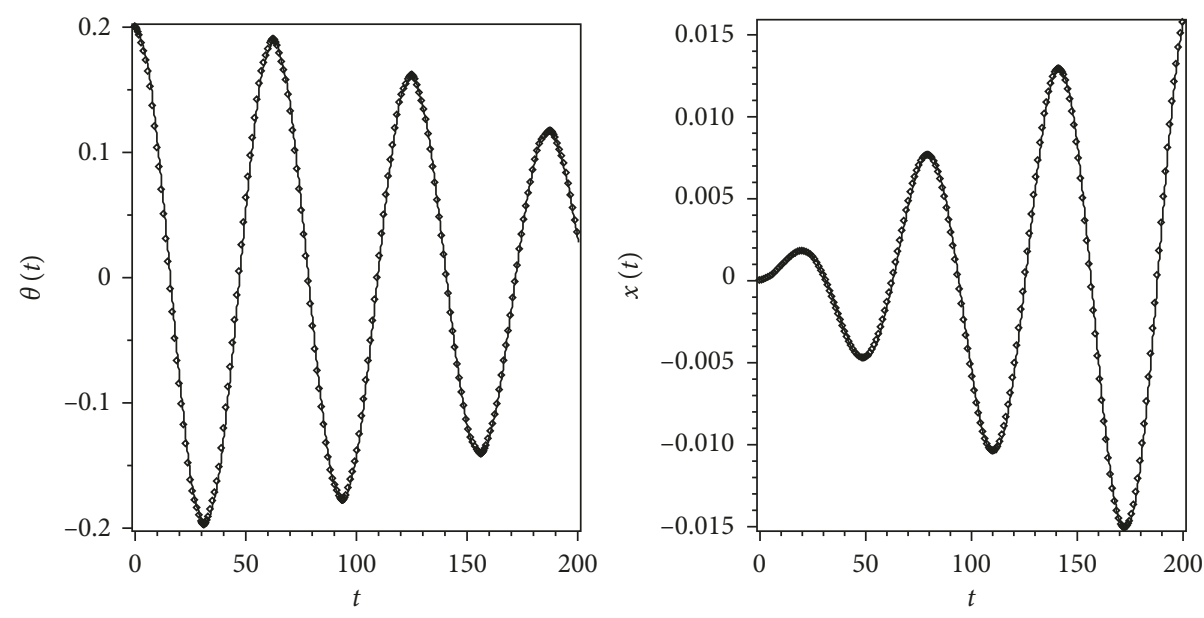

(a)
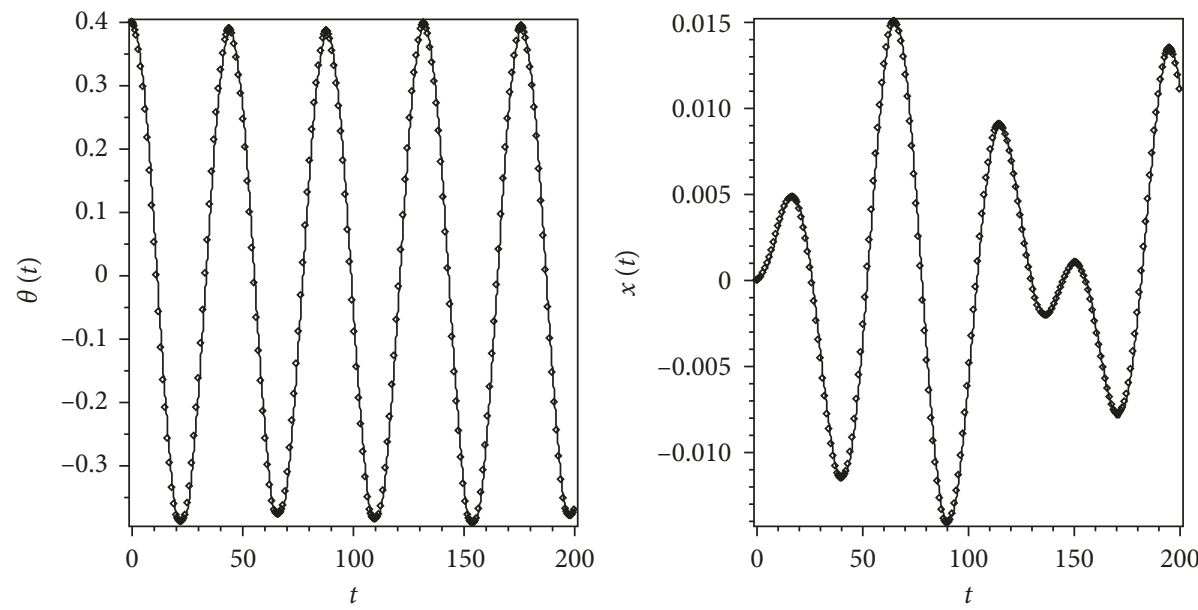

(b)

FIGURE 2: Comparison between the analytical approximation (dot) and the numerical solution (solid line) of Equation (6) with $\beta_{1}=1, \beta_{2}=0.01$, and $\beta_{3}=0.01$, and the initial conditions are taken as $\theta(0)=0.2, \theta(0)=0, x(0)=0$, and $\dot{x}(0)=0:(\mathrm{a}) \epsilon=0.01, \delta=0.01 ;(\mathrm{b}) \epsilon=0.01, \delta=0.02$.
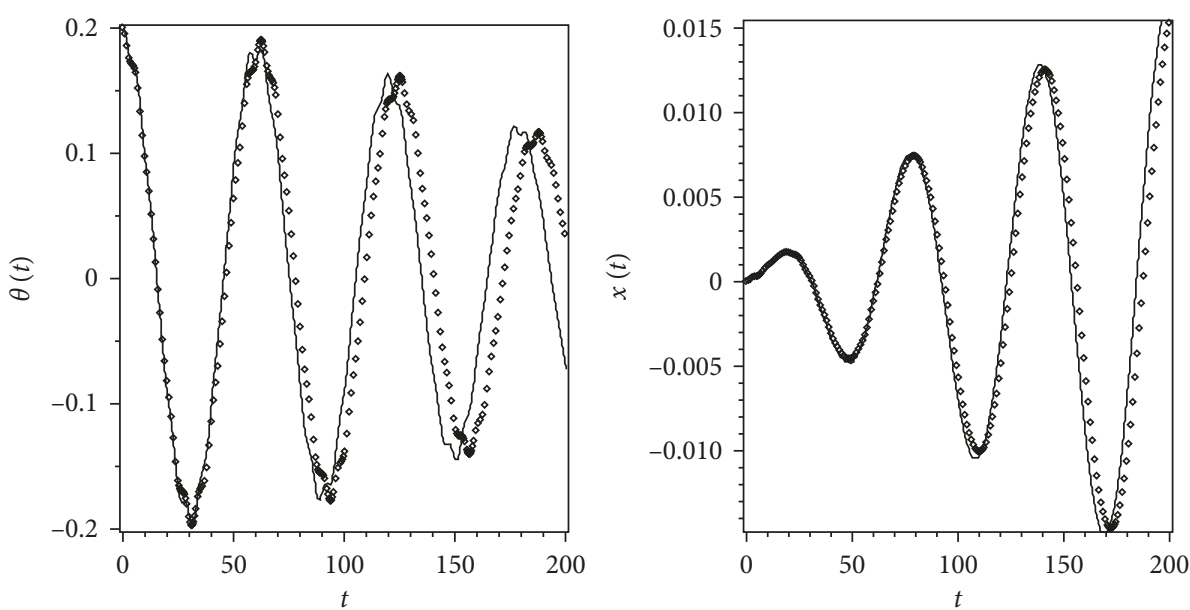

(a)

Figure 3: Continued. 

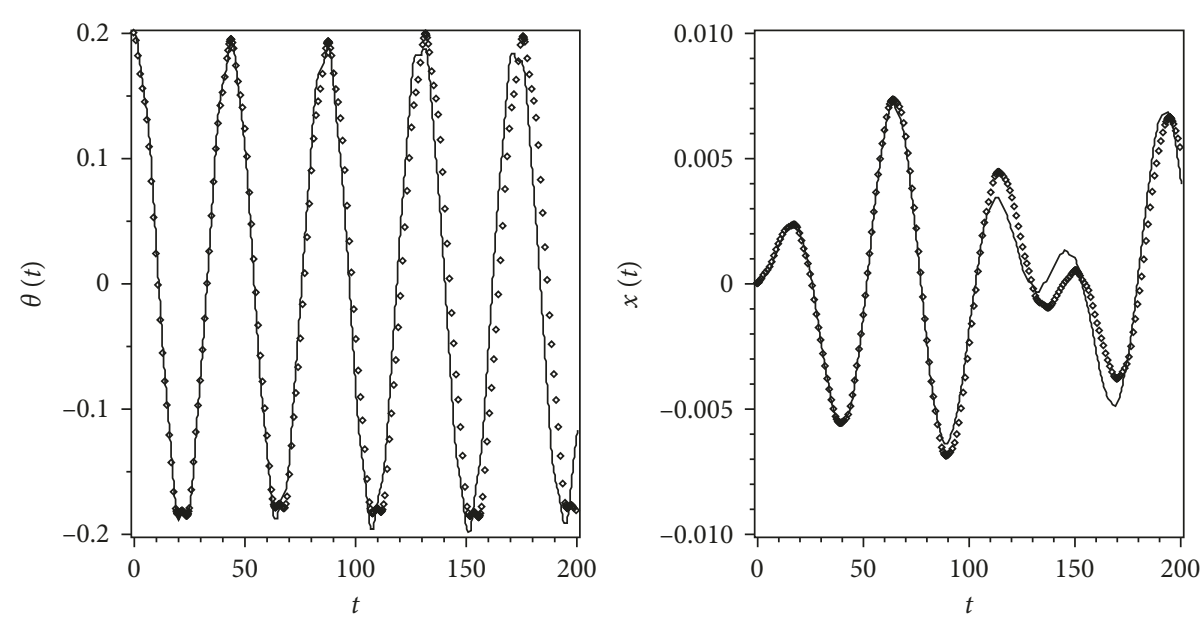

(b)

FIGURE 3: Comparison between the analytical approximation (dot) and the numerical solution (solid line) of Equation (6) with $\beta_{1}=1, \beta_{2}=0.01$, $\beta_{3}=0.01$, and the initial conditions are taken as $\theta(0)=0.2, \dot{\theta}(0)=0, x(0)=0$, and $\dot{x}(0)=0$ : (a) $\epsilon=0.04, \delta=0.01 ;(\mathrm{b}) \epsilon=0.04, \delta=0.02$.

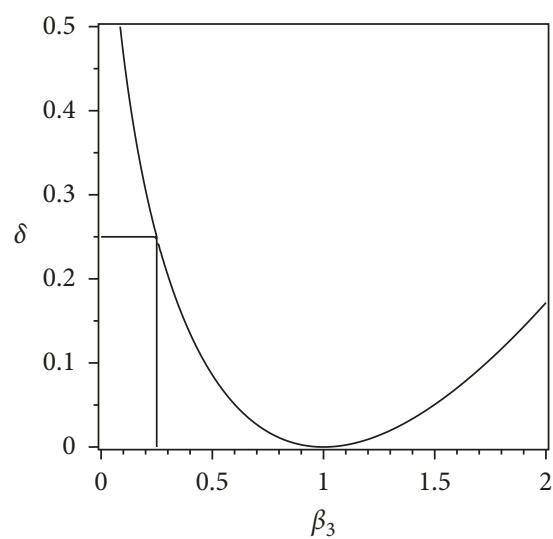

(a)

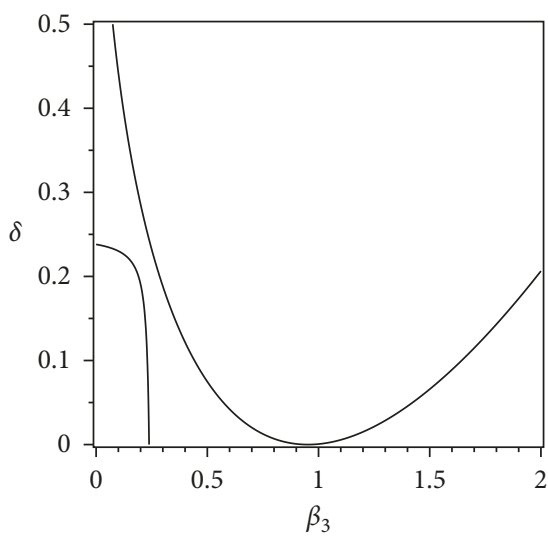

(c)

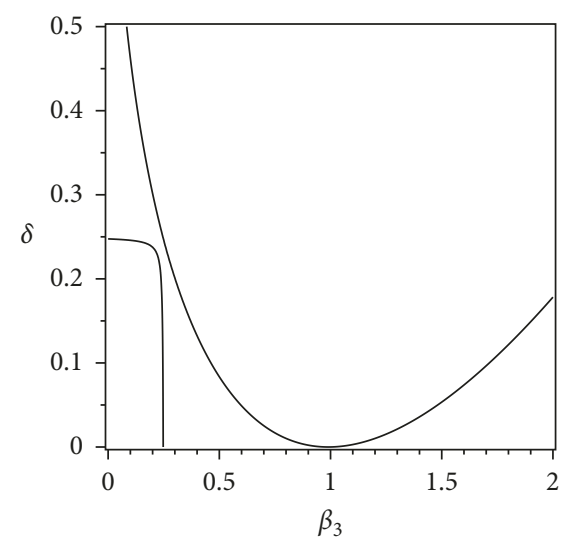

(b)

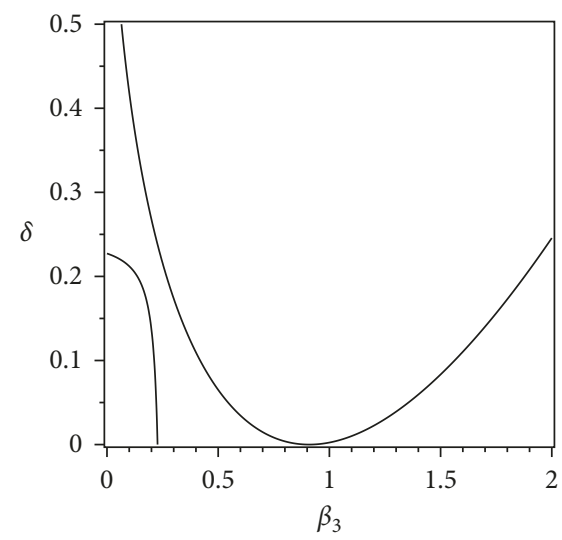

(d)

FIgURe 4: The critical curves defined by Equation (17): (a) $n=0$; (b) $n=0.01$ (c) $n=0.05$; (d) $n=0.1$.

Our theoretical analysis indicates that excessive lateral vibrations of the bridge occur if the lateral natural frequency of the bridge near half the vertical step frequency of the pedestrians. On the other hand, the lateral step frequency is also half the vertical step frequency for a normal pedestrian, and the ratio is almost unaffected even if the pedestrian walks on a moving surface $[5,16]$. For a footbridge with a lateral natural frequency between $0.75-1.25 \mathrm{~Hz}$, once 


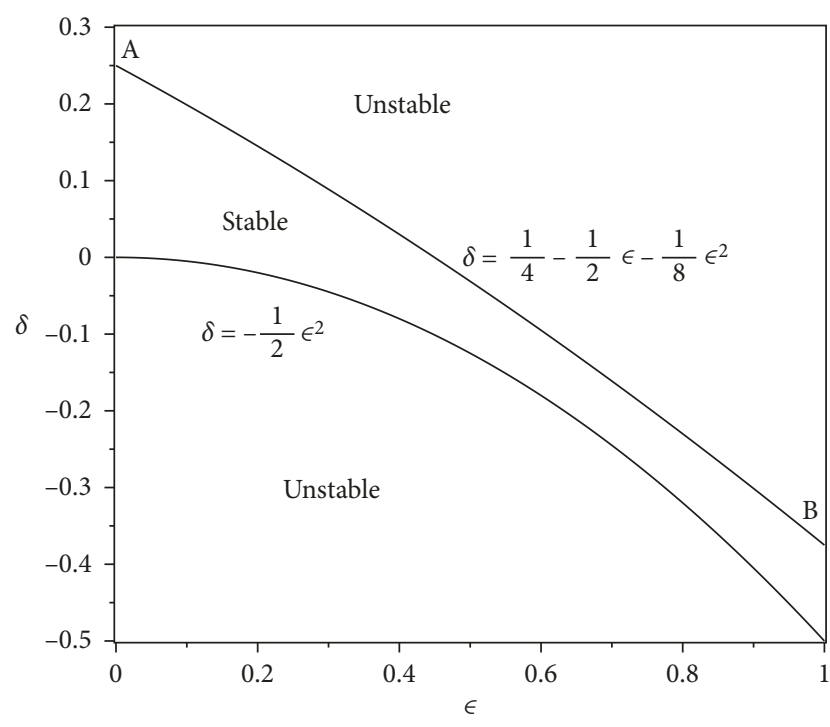

FIgURE 5: The transition curves in the $\epsilon-\delta$ plane.

pedestrians walk on the bridge, resonance will be inevitable. In fact, excessive lateral vibrations are easily observed in footbridges with a lateral natural frequency near $1 \mathrm{~Hz}$; for example, the lateral natural frequencies of the T-bridge, the M-bridge, and the London Millennium bridge are between 0.9-1.1 Hz [2]. Since the ratio between the lateral and vertical step frequencies remains unchanged despite a small or large lateral amplitude of the bridge, frequency-locking phenomenon is easy to be explained. To deeply understand this in theory, we discuss the stability of the inverted pendulum system near $\sqrt{\beta_{3}}=1 / 2$ in the next section.

\section{Stability Analysis of the Inverted Pendulum System}

In the section, we investigate the stability of solutions of Equation (6) with $-1 \ll \delta<0$. We consider that both the initial displacement and velocity of the bridge are zero. Assume that $\theta$ and $x$ can be written as

$$
\begin{aligned}
& \theta=\theta_{0}+\beta_{2} \theta_{1}+\cdots, \\
& x=x_{0}+\beta_{2} x_{1}+\cdots .
\end{aligned}
$$

Substituting Equation (21) into Equation (6) and equating the coefficients of each power of $\beta_{2}$ to be zero, we have

$$
\begin{aligned}
\ddot{\theta}_{0}+(\delta+\epsilon \cos (t)) \theta_{0} & =-\beta_{1} \ddot{x}_{0}, \\
\ddot{x}_{0}+\beta_{3} x_{0} & =0, \\
\ddot{\theta}_{1}+(\delta+\epsilon \cos (t)) \theta_{1} & =-\beta_{1} \ddot{x}_{1}, \\
\ddot{x}_{1}+\beta_{3} x_{1} & =-\ddot{\theta}_{0} .
\end{aligned}
$$

Equation (6) has stable solutions only if the solutions of both Equations (22) and (23) are stable. To obtain the stability conditions of solutions of Equation (6), we separately analyze the stability of solutions of Equations (22) and (23).
4.1. The Stability Conditions of Solutions of Equation (22). Since the initial displacement and velocity of the bridge are zero, the solution of the second equation in Equation (22) is $x_{0}=0$. Substituting such solution into the first equation in Equation (22) yields

$$
\ddot{\theta}_{0}+(\delta+\epsilon \cos (t)) \theta_{0}=0
$$

Equation (24) is a Mathieu equation without external force. Since the stability results of Equation (24) are well known, we omit the analysis process here. The two transition curves emanating from the origin and $\delta=1 / 4$ and corresponding periodic solutions are separately given by (please see the reference $[14,15]$ for details)

$$
\begin{aligned}
\delta= & -\frac{1}{2} \epsilon^{2}, \\
\theta_{0}= & a\left[1+\epsilon \cos t+\frac{1}{8} \epsilon^{2}(\cos (2 t)-1)\right], \\
\delta= & \frac{1}{4}-\frac{1}{2} \epsilon-\frac{1}{8} \epsilon^{2}, \quad \delta<0 \\
\theta_{0}= & a \cos \left(\frac{1}{2} t\right)+\frac{1}{4} \epsilon \cos \left(\frac{3}{2} t\right) \\
& -\frac{1}{48} \epsilon^{2}\left[3 \cos \left(\frac{3}{2} t\right)-\cos \left(\frac{5}{2} t\right)\right],
\end{aligned}
$$

where $a$ is a constant determined by the initial conditions. Equations (25) and (26) are obtained by using the perturbation method. The stability chart presented in Figure 5 is depicted based on Equations (25) and (26). It should be noted that Equations (25) and (26) only are valid for small $\epsilon$. Once $\epsilon$ is not small, the results obtained by perturbation analysis might lose accuracy. At $\epsilon=0.5$, the expression (26) yields $\delta=-0.03125$. The exact value is $\delta=-0.02756$. From the analysis results in literature [14], the approximate expression $\delta=1 / 4-0.556$ is valid over a large range of $\epsilon$. Considering physical meanings of $\delta$ and $\epsilon$, we focus on the region $\delta \epsilon(-0.15,0)$. Therefore, the precision of Equation (26) is enough in the study of the inverted pendulum system in this paper.

4.2. The Stability Conditions of Solutions of Equation (23). Since the ratio between the lateral and vertical step frequencies is $1 / 2$ for a pedestrian, we substitute Equation (26) into the second equation in Equation (23):

$$
\begin{aligned}
\ddot{x}_{1}+\beta_{3} x_{1}= & \frac{a}{4} \cos \left(\frac{1}{2} t\right)+\frac{9 a \epsilon}{64}(4-\epsilon) \cos \left(\frac{3}{2} t\right) \\
& +\frac{25 a \epsilon^{2}}{192} \cos \left(\frac{5}{2} t\right) .
\end{aligned}
$$

According to the engineering practice and the analysis in previous section, we focus on the case of $\beta_{3} \neq 1 / 4$ and $\beta_{3}$ near 1/4. The particular solution of Equation (27) is written as

$$
x_{1}=a q_{1} \cos \left(\frac{1}{2} t\right)+a q_{2} \cos \left(\frac{3}{2} t\right)+a q_{3} \cos \left(\frac{5}{2} t\right),
$$


where

$$
\begin{aligned}
& q_{1}=\frac{1}{4\left(4 \beta_{3}-1\right)}, \\
& q_{2}=\frac{81 \epsilon(4-\epsilon)}{64\left(4 \beta_{3}-9\right)}, \\
& q_{3}=\frac{625 \epsilon^{2}}{192\left(4 \beta_{3}-25\right)} .
\end{aligned}
$$

Substituting Equation (28) into the first equation in Equation (23), we have

$$
\begin{aligned}
\ddot{\theta}_{1}+(\delta+\epsilon \cos (t)) \theta_{1}= & q_{4} \cos \left(\frac{1}{2} t\right)+q_{5} \cos \left(\frac{3}{2} t\right) \\
& +q_{6} \cos \left(\frac{5}{2} t\right),
\end{aligned}
$$

where

$$
\begin{aligned}
& q_{4}=\frac{1}{4} \beta_{1} a q_{1}, \\
& q_{5}=\frac{9}{4} \beta_{1} a q_{2}, \\
& q_{6}=\frac{25}{4} \beta_{1} a q_{3} .
\end{aligned}
$$

Similar to that treated in the previous section, we need to analyze the stability condition of periodic solutions with period of $4 \pi$ in Equation (30). Considering the linearity of Equation (30), we focus on the following equation:

$$
\ddot{z}+(\delta+\epsilon \cos (t)) z=q \cos (w t),
$$

where $q=q_{4}$ and $w=1 / 2$. In the following, we use the energy method [17] to analyze the condition that Equation (32) with $\delta>0$ has a stable periodic solution with period of $4 \pi$. Note that the stability condition will be still valid for the case of $\delta<0$ according to the research conclusion for the Mathieu equation. We denote two functions:

$$
\begin{aligned}
g(z) & =\delta z, \\
f(z, \dot{z}, t) & =\epsilon \cos (t) z-q \cos (w t) .
\end{aligned}
$$

The potential energy and the mechanical energy of system (32) are expressed by

$$
\begin{aligned}
V(z) & =\int_{0}^{z} g(y) d y=\frac{1}{2} \delta z^{2}, \\
E(z, \dot{z}, t) & =V(z)+\frac{1}{2} \dot{z}^{2} .
\end{aligned}
$$

The energy coordinate system $(E, \psi)$ corresponding to system (32) is obtained by the following transformation:

$$
\begin{aligned}
& z=a \cos (\psi), \\
& \dot{z}=-\sqrt{2(V(a)-V(a \cos (\psi)))}=-a \sqrt{\delta} \sin (\psi),
\end{aligned}
$$

where the terms higher than the second order are neglected. Additionally,

$$
z g(z)=\delta z^{2}>0, \quad \text { if } z \neq 0 .
$$

According to Equation (35), system (32) is transformed into

$$
\begin{aligned}
\frac{d E}{d t}= & -f(z, \dot{z}, t) \dot{z} \\
= & \frac{a^{2} \epsilon \sqrt{\delta}}{4}[\sin (2 \psi-t)+\sin (2 \psi+t)] \\
& -\frac{q a \sqrt{\delta}}{2}[\sin (\psi+w t)+\sin (\psi-w t)], \\
\frac{d \psi}{d t}= & \frac{1}{a \sin (\psi)}\left(\frac{1}{2 g(-a) g(a)}[(g(-a)-g(a)) \cos (\psi)\right. \\
& \left.+(g(-a)+g(a))] \frac{d E}{d t}-\dot{z}\right) \\
= & \sqrt{\delta}+\frac{\epsilon}{4 \sqrt{\delta}}[2 \cos (t)+\cos (2 \psi+t)+\cos (2 \psi-t)] \\
& -\frac{q}{2 a \sqrt{\delta}}[\cos (\psi+w t)+\cos (\psi-w t)] .
\end{aligned}
$$

Since we assume that Equation (32) has a periodic solution with period of $4 \pi$, the phase $\psi$ can be written as

$$
\psi=\frac{1}{2} t+\phi
$$

which can be transformed into the following form:

$$
t=2(\psi-\phi) \text {. }
$$

Substituting Equation (39) into Equation (37) leads to

$$
\begin{aligned}
\frac{d E}{d t}= & \frac{a^{2} \epsilon \sqrt{\delta}}{4}[\sin (2 \phi)+\sin (2(2 \psi-\phi))] \\
& -\frac{q a \sqrt{\delta}}{2}[\sin ((2 w+1) \psi-2 w \phi)+\sin ((1-2 w) \psi+2 w \phi)],
\end{aligned}
$$

$\frac{d \psi}{d t}=\sqrt{\delta}+\frac{\epsilon}{4 \sqrt{\delta}}[2 \cos (2(\psi-\phi))+\cos (2(2 \psi-\phi))+\cos (2 \phi)]$

$$
-\frac{q}{2 a \sqrt{\delta}}[\cos ((1+2 w) \psi-2 w \phi)+\cos ((1-2 w) \psi+2 w \phi)] .
$$

Considering $w=1 / 2$ in Equation (40), the existence of periodic solutions with period of $4 \pi$ means that the following equations should be held:

$$
\begin{gathered}
\frac{a^{2} \epsilon \sqrt{\delta}}{4} \sin (2 \phi)-\frac{\beta_{1} a^{2} q_{1} \sqrt{\delta}}{8} \sin (\phi)=0, \\
\sqrt{\delta}+\frac{\epsilon}{4 \sqrt{\delta}} \cos (2 \phi)-\frac{\beta_{1} q_{1}}{8 \sqrt{\delta}} \cos (\phi)=\frac{1}{2} .
\end{gathered}
$$

Solving the first equation of Equation (41), we have 


$$
\begin{aligned}
a & =\text { constant }, \\
\cos (\phi) & =\frac{q_{1} \beta_{1}}{4}, \\
\phi & =0, \\
\phi & =\pi .
\end{aligned}
$$

Then, the second equation of Equation (41) becomes

$$
\begin{aligned}
\cos (\phi) & =\frac{q_{1} \beta_{1}}{4}: \quad 4 \delta-\epsilon-2=0, \\
\phi & =0: \quad 4 \delta-\epsilon-2=\frac{q_{1} \beta_{1}}{2}, \\
\phi & =\pi: \quad 4 \delta-\epsilon-2=-\frac{q_{1} \beta_{1}}{2} .
\end{aligned}
$$

Considering condition (26) and the first equation in Equation (43), we have $\epsilon=-5.646$ or $\epsilon=-0.3542$, which is not possible in the practical engineering. Recalling $\epsilon=A / L$, $\beta_{1}=1 / L$, and $q_{1}=1 /\left[4\left(4 \beta_{3}-1\right)\right]$, the last two equations in Equation (43) can be rewritten as

$$
\begin{aligned}
& \phi=0: \quad \beta_{3}=\frac{8 L^{2}+(24 A-1) L+4 A^{2}}{16\left(A^{2}+6 A L+2 L^{2}\right)}, \\
& \phi=\pi: \quad \beta_{3}=\frac{8 L^{2}+(24 A+1) L+4 A^{2}}{16\left(A^{2}+6 A L+2 L^{2}\right)} .
\end{aligned}
$$

According to stability analysis for Equation (23), if the periodic motion of the pedestrians has a period of $4 \pi$, condition (44) must be satisfied. We show such stability condition in Figure 6. Clearly, $\beta_{3}$ approaches to $1 / 4$ with the increase of $A$ for a fixed $L$ (Figure 6(a)) or with the increase of $L$ for a fixed $A$ (Figure 6(b)).

From the stability analysis for Equations (22) and (23), the pedestrians on the bridge are indeed caught in a dilemma. For the given body height and vertical step frequency, the pedestrians have to increase the vertical vibration amplitude as much as possible to habitually keep the ratio of $1 / 2$ between the lateral and vertical step frequencies, which just increases the risk of resonance of the bridge. Since the walking habit is hard to be changed, the occurrence of the excessive lateral vibrations of the bridge is inevitable as long as the lateral natural frequency of the bridge approaches half the vertical step frequency of the pedestrians (about in the range of $0.75-1.25 \mathrm{~Hz})$.

\section{Numerical Simulations}

To demonstrate that the proposed inverted pendulum model in this paper can give quantitative results, we carry out numerical simulations for Equation (6) with damping:

$$
\ddot{\theta}+\beta_{1} \ddot{x}+(\epsilon \cos (t)+\delta) \theta=0, \ddot{x}+\beta_{2} \ddot{\theta}+\beta_{3} x+c \dot{x}=0,
$$

where $c=C /(M+m)$, in which $C$ is the damping coefficient, and $\beta_{1,2,3}$ is defined in Equation (6). We adopt the parameters of the M-bridge in Japan [3], and the following three cases will be considered in this section:

(i) Case of T-1 (symmetric first): lateral natural frequency is $0.9 \mathrm{~Hz} M=2.14 e 5, m=1.4969 e 4, k=$ 7.307361e6, and $C=2.8262 e 4$

(ii) Case of M-1 (symmetric fourth): lateral natural frequency is $1.025 \mathrm{~Hz} M=9.72 e 4, m=3.35 e 3, k=$ 4.031566e6, and $C=3.387 e 3$

(iii) Case of M-4 (asymmetric third): lateral natural frequency is $0.879 \mathrm{~Hz} M=9.72 e 4, m=2.469 e 3$, $k=2.964857 e 6$, and $C=2.905 e 3$

According to theoretical analysis in previous two sections, resonance occurs when the lateral vertical frequency is close to the twice lateral natural frequency of the bridge. For the case of T-1, we choose $A=0.576, \omega=2 \pi \times 1.6$, and $A=0.57163, \omega=2 \pi \times 1.89$, respectively. The timehistory curves of $x$ and $\theta$ are given in Figure 7. The natural frequency of the bridge is $0.9 \mathrm{~Hz}$, and resonance occurs near $\omega=2 \pi \times 0.9$. Then, the maximum response should be close to $15 \mathrm{~mm}$. The maximum measured response for the case of T-1 is $13.3 \mathrm{~mm}$. In addition, comparing Figures 7(a) with $7(\mathrm{~b})$, it is clear that the swing angle of pedestrians increases from $0.01 \mathrm{rad}$ to $0.06 \mathrm{rad}$, and the vibration amplitude of the bridge increases from $1.5 \mathrm{~mm}$ to $15 \mathrm{~mm}$ when the vertical step frequency increases from $1.6 \mathrm{~Hz}$ to $1.89 \mathrm{~Hz}$. There is a dramatic increase when the vertical step frequency approaches to twice the natural frequency of the bridge.

For the case of $\mathrm{M}-1$, we separately choose $A=0.559$, $\omega=2 \pi \times 1.75$, and $A=0.505, \omega=2 \pi \times 2.01$ to calculate the time-history curves of system (45), which are shown in Figure 8 . When the vertical step frequency approaches to twice the natural frequency of the bridge $1.025 \mathrm{~Hz}$, the vibration amplitude of the bridge increases rapidly, while the swing angle of the pedestrians is not very large and increases slowly. According to [3], the measured response for the case of M-1 is $40.1 \mathrm{~mm}$. Finally, we take $A=0.549, \omega=2 \pi \times 1.7$, and $A=0.51, \omega=2 \pi \times 2.04$, respectively, to numerically obtain the time-history curves of system (45) for case of M-4. The results are presented in Figure 9, which are similar to Figure 8 . The measured response for the case of M-4 is $34.8 \mathrm{~mm}$. The numerical simulations presented in this section verify that the presented inverted pendulum model can predict effectively responses for the M-bridge. And excessive lateral vibrations occur when the vertical step frequency of pedestrians approach to the lateral natural frequency of a bridge.

\section{Conclusions}

In this paper, we use the inverted pendulum on a cart to study the lateral interaction between synchronization pedestrians and a footbridge. The pivot point of the inverted pendulum on the cart is considered to vertically vibrate to model the synchronization pedestrians' walking motion. The proposed model avoids the difficulty of the determination of lateral force induced by synchronization pedestrians on the 


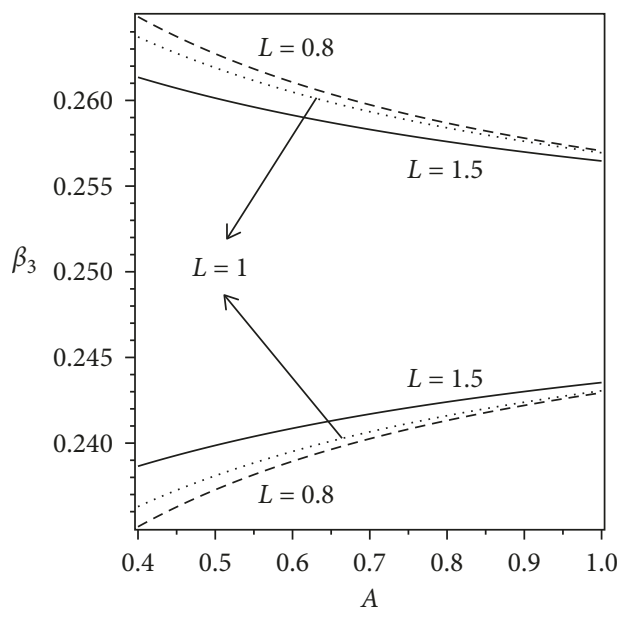

(a)

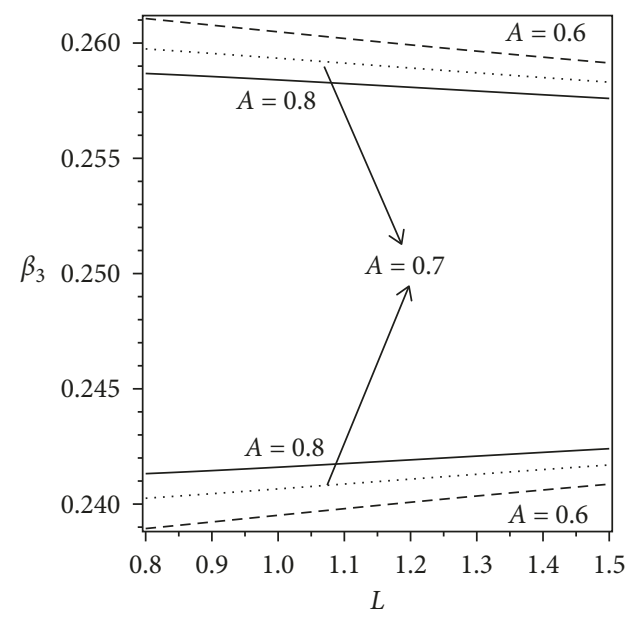

(b)

Figure 6: The conditions (44) that pedestrians on the bridge sways with period of $4 \pi$ : (a) in the $A-\beta_{3}$ plane for $L=0.8,1$, and 1.5 ; (b) in the $L-\beta_{3}$ plane for $A=0.6,0.7$, and 0.8 .
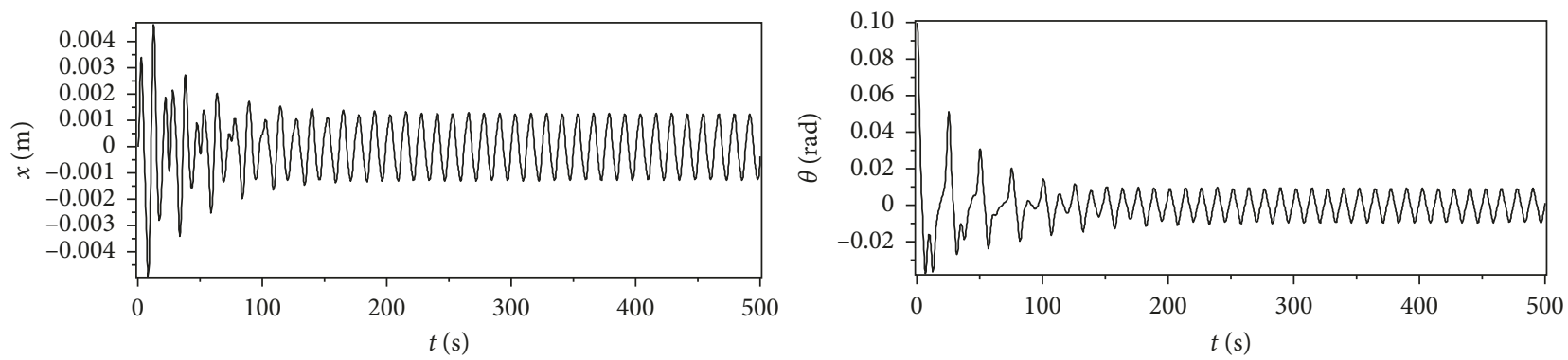

(a)
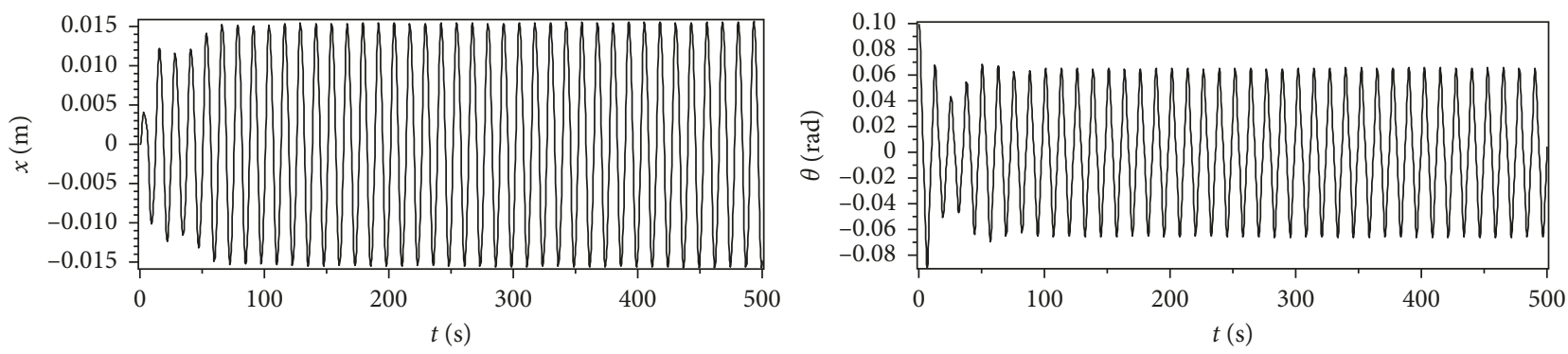

(b)

Figure 7: The time-history curves of system (45) for the case of T-1: (a) $A=0.576, \omega=2 \pi \times 1.6$; (b) $A=0.57163, \omega=2 \pi \times 1.89$.

bridge, which always was treated semiempirically in previous researches. The governing equation of the inverted pendulum system reduces to the classical Mathieu equation if the mass of the cart is sufficiently large, and thus it can be analytically investigated by using the perturbation method and energy method.

The analysis of the inverted pendulum model shows that the most probable resonance condition for the lateral vibrations of the bridge is that the lateral natural frequency of the bridge is half the vertical step frequency of the synchronization pedestrians. Unfortunately, pedestrians have the walking habit of keeping the ratio of $1 / 2$ between the lateral and vertical step frequencies. For a given body height and a vertical step frequency, a pedestrian needs to increase the vertical vibration amplitude of the body as much as possible to keep the ratio of $1 / 2$ between the lateral and vertical step frequencies under the conditions of body balance. However, the larger the vertical vibration amplitude of the body, the greater the risk of the resonance of the bridge. Once the lateral natural frequency of the bridge is in the range of $0.75-1.25 \mathrm{~Hz}$, the resonance occurs inevitable. Our analysis shows that the walking habit keeping the ratio of $1 / 2$ between the lateral and vertical step frequencies is the key cause of frequency-locking phenomenon. In addition, 

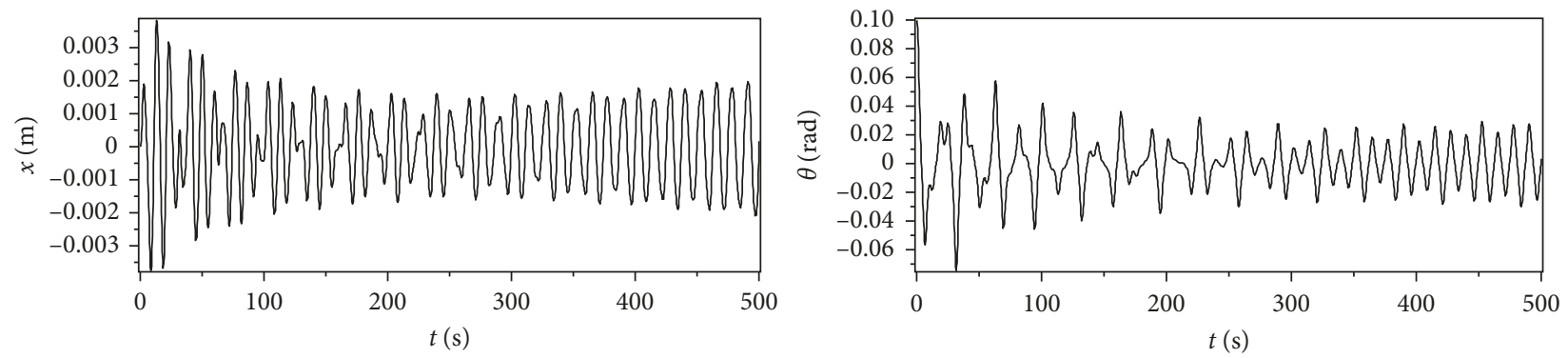

(a)
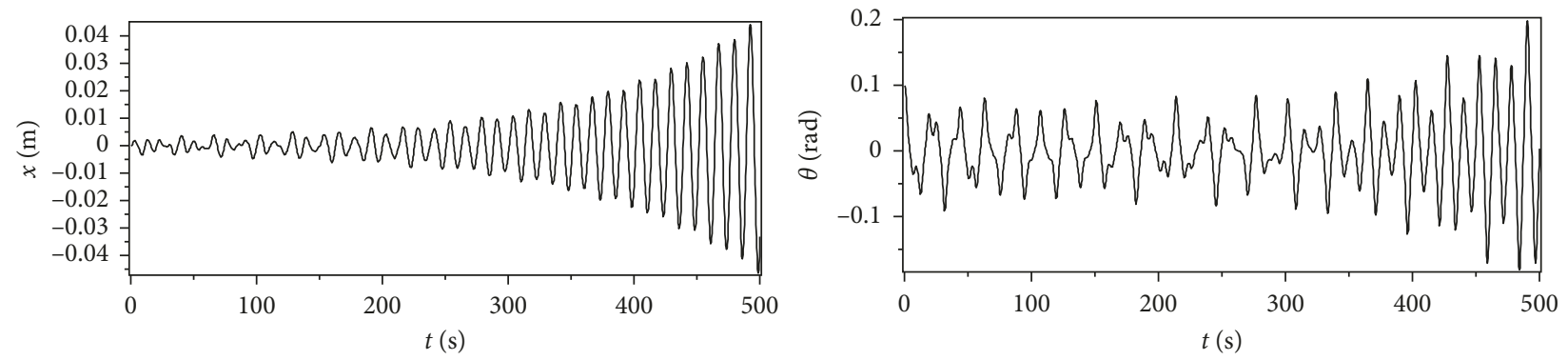

(b)

Figure 8: The time-history curves of system (45) for the case of M-1: (a) $A=0.559, \omega=2 \pi \times 1.75$; (b) $A=0.505, \omega=2 \pi \times 2.01$.
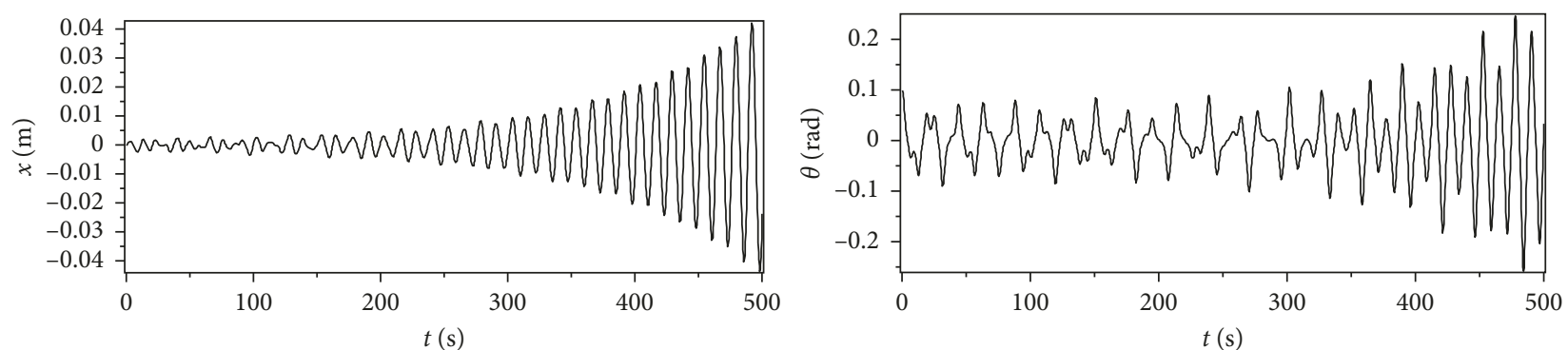

(a)
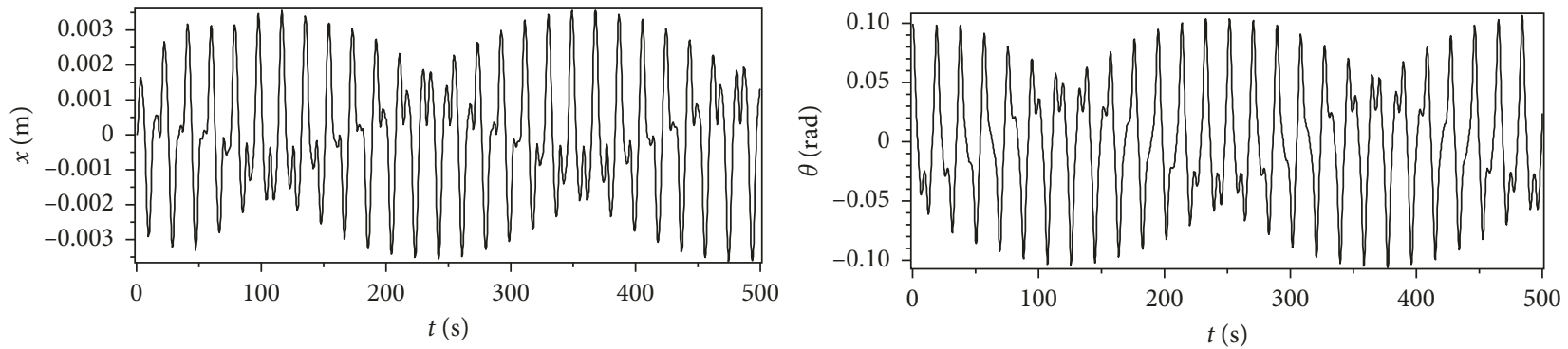

(b)

Figure 9: The time-history curves of system (45) for the case of M-4: (a) $A=0.549, \omega=2 \pi \times 1.7$; (b) $A=0.51, \omega=2 \pi \times 2.04$.

the analysis results can be used to explain why excessive lateral vibrations are often observed in many footbridges with a lateral natural frequency of about $1 \mathrm{~Hz}$, such as Tbridge, M-bridge in Japan, and the London Millennium Bridge.

The analysis results also can be used to explain the famous "jump phenomenon" in the Millennium Bridge. The bridge began to laterally vibrate with a large amplitude once the number of pedestrians is greater than the critical one.
However, it was found that the lateral amplitude of the bridge rapidly decreased while the pedestrians did not significantly reduce their walking speed. In our opinion, excessive lateral vibrations of the bridge make the pedestrians feel uncomfortable, and the pedestrians did not change their walking speed but instinctively adjusted their vertical step frequencies. Then, the ratio between the lateral natural frequency of the bridge and the vertical step frequency was far away from the value of $1 / 2$. On the other 
hand, the ratio of $1 / 2$ between the lateral and vertical step frequencies still remained the same, and then the frequency of the lateral force induced by pedestrians deviated from the range of $0.75-1.25 \mathrm{~Hz}$, which destroyed the resonance conditions and eventually resulted in the dramatic reduction of the lateral amplitude sof the bridge.

\section{Data Availability}

The data used to support the findings of this study are included within the article.

\section{Conflicts of Interest}

The authors declare that there are no conflicts of interest regarding the publication of this paper.

\section{Authors' Contributions}

All authors carried out the proofreading of the paper. All authors conceived the study and participated in its design and coordination. All authors read and approved the final paper.

\section{Acknowledgments}

The authors appreciate very much the advices of the reviewers. The advices will benefit the improvement of the paper and our future research. This work was supported by the National Natural Science Foundation of China (Grant nos. 11472160, 11672177, and 11672185) and Jiangxi Natural Science Foundation (Grant no. 20161BAB216103).

\section{References}

[1] H. Bachmann, A. J. Pretlove, and H. Rainer, "Dynamic forces from rhythmical human body motions," in Vibration Problems in Structure: Practical Guidelines, Birkhauser, Basel, Switzerland, 1995.

[2] S. Nakamura, "Model for lateral excitation of footbridges by synchronous walking," Journal Structural Engineering ASCE, vol. 130, no. 1, pp. 32-37, 2004.

[3] S. Nakamura and T. Kawasaki, "A method for predicting the lateral girder response of footbridges induced by pedestrians," Journal of Constructional Steel Research, vol. 65, no. 8-9, pp. 1705-1711, 2009.

[4] Y. Fujino, P. Pacheco, S. Nakamura, and P. Warnitchai, "Synchronization of human walking observed during lateral vibration of a congested pedestrian bridge," Earthquake Engineering and Structural Dynamical, vol. 22, no. 9, pp. 741758, 1993.

[5] G. Piccardo and F. Tubino, "Parametric resonance of flexible footbridges under crowd-induced lateral excitation," Journal of Sound and Vibration, vol. 311, no. 1-2, pp. 353-371, 2008.

[6] P. Dallard, A. J. Fitezpatrick, A. Flint et al., "The London Millennium footbridge," The Structural Engineer, vol. 79, pp. 17-33, 2001.

[7] T. M. Roberts, "Lateral pedestrian excitation of footbridges," Journal of Bridge Engineering ASCE, vol. 10, no. 1, pp. 107-112, 2005.
[8] J. H. G. Macdonald, "Lateral excitation of bridge by balancing pedestrians," Proceedings of the Royal Society A: Mathematical, Physical and Engineering Sciences, vol. 465, no. 2104, pp. 1055-1073, 2008.

[9] Y. Fujino and D. M. Siringoringo, "A conceptual review of pedestrian-induced lateral vibaration and crowd synchronization problem on footbridges," Journal of Bridge Engineering, vol. 21, no. 8, pp. C4015001-C4015012, 2016.

[10] Y. Matsumoto, T. Nishioka, H. Shiojiri, and K. Matsuzaki, "Dynamic design of footbridges," in Proceedings of IABSE, pp. 1-15, Zürich, Switzerland, 1978.

[11] E. T. Ingólfsson, C. T. Georgakis, F. Ricciardelli, and J. Jönsson, "Experimental identification of pedestrian-induced lateral forces on footbridges," Journal of Sound and Vibration, vol. 330, no. 6, pp. 1265-1284, 2011.

[12] A. N. Blekherman, "Swaying of pedestrian bridges," Journal of Bridge Engineering ASCE, vol. 10, no. 2, pp. 142-150, 2005.

[13] J. A. Blackburn, H. J. T. Smith, and N. Gronbech-Jensen, "Stability and Hopf bifurcations in an inverted pendulum," American Journal of Physics, vol. 60, no. 10, pp. 903-908, 1992.

[14] N. W. McLanchlan, Theory and Application of Mathieu Functions, Oxford University Press, New York, NY, USA, 1951.

[15] J. J. Stoker, Nonlinear Vibrations in Mechanical and Electrical Systems, Wiley/Interscience, New York, NY, USA, 1966.

[16] F. Tubino and G. Piccardo, "A loading model for the interpretation of lateral vibrations of flexible footbridges," in Proceedings of Fourth International Footbridge 2011 Conference, Wroclaw, Poland, July 2011.

[17] L. Li and Y. Hongling, "Energy method for computing periodic solutions of strongly nonlinear autonomous systems with multi-degree-of-freedom," Nonlinear Dynamics, vol. 31, no. 1, pp. 23-47, 2003. 


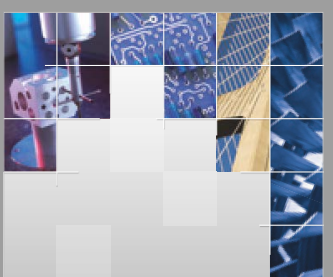

\section{Enfincering}
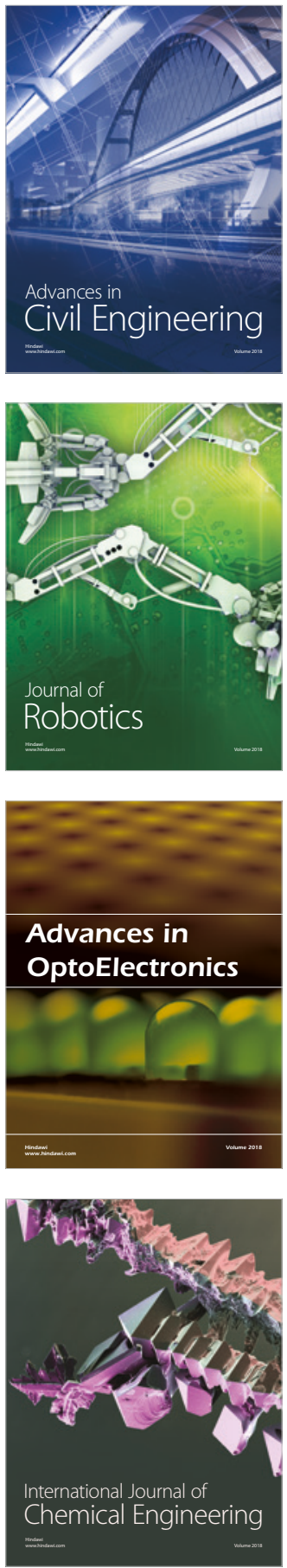

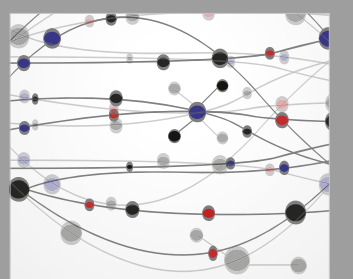

\section{Rotating \\ Machinery}

The Scientific World Journal

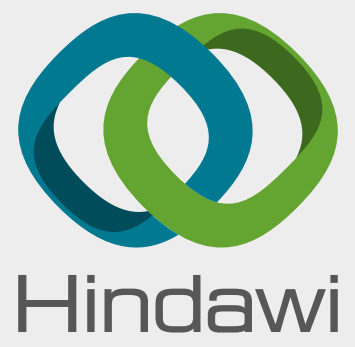

Submit your manuscripts at

www.hindawi.com
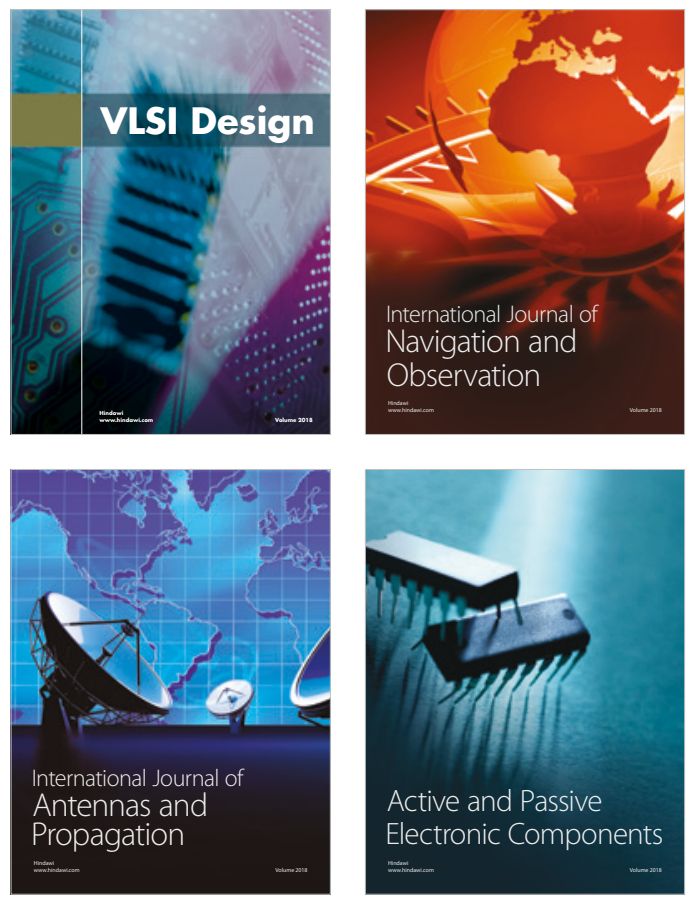
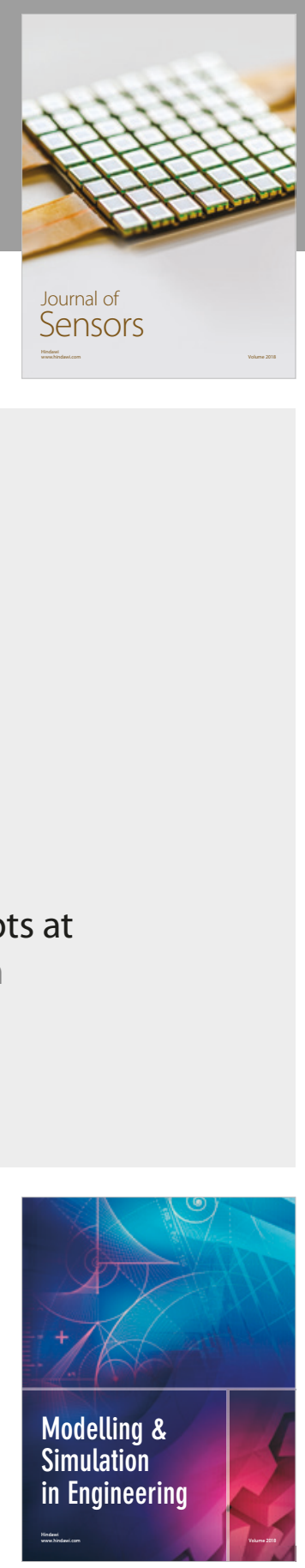

\section{Advances \\ Multimedia}
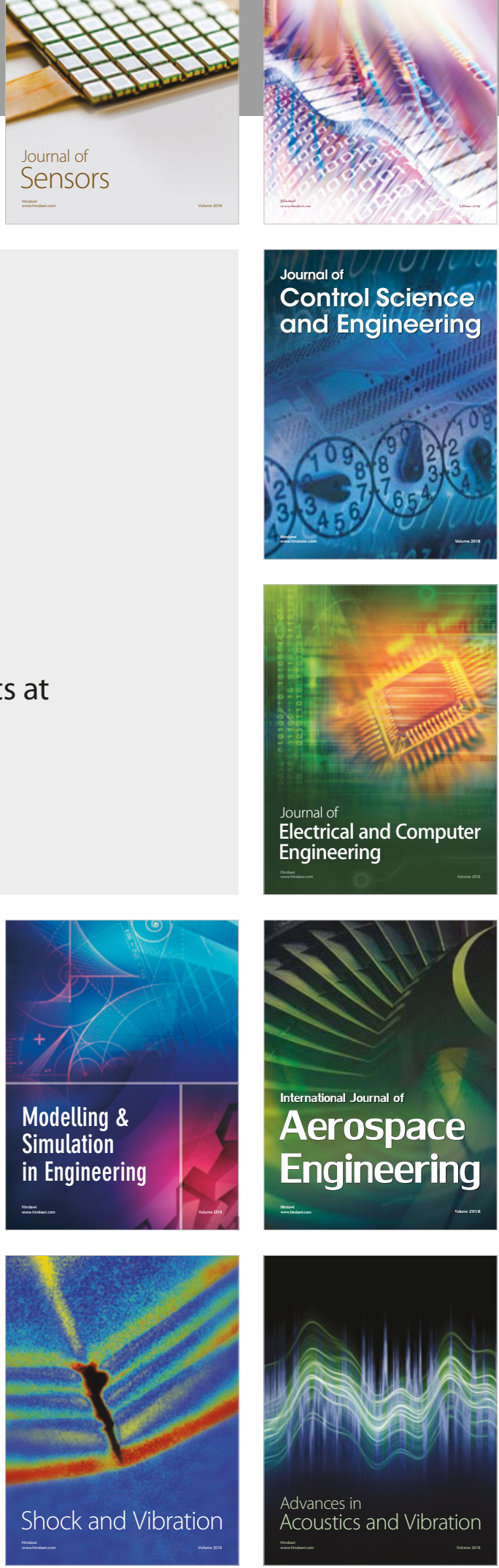